\title{
A Numerical Simulation of Turbulence Flow around a Blade Profile of HAWT Rotor in Moving Pulse
}

\author{
N. Belkheir ${ }^{1}$, R. Dizene ${ }^{2 \dagger}$ and S. Khelladi ${ }^{3}$ \\ ${ }^{1}$ Research assistant, university center of Khemis Milia, Ain Defla Algeria \\ ${ }^{2}$ Professor, USTHB university of Algiers; POBox 32 El Alia, Algiers Algeria \\ ${ }^{3}$ Maitre de Conférences au LEMFI, Arts \& Métiers Paris Tech, France
}

$\dagger$ Corresponding Author Email: rd_2006diz.anis@yahoo.fr

(Received December 11, 2009; accepted February 29, 2010)

\begin{abstract}
A computation of three-dimensional flow past an isolated NACA0012 airfoil using Reynolds-averaged Navier -stokes equations (RANS) method is conducted. Tow closure models k- $\varepsilon$ and SST are applied to the numerical prediction of the turbulent flow. A two-dimensional numerical simulation of oscillatory movement of the profile, based on solving URANS equations is then studied for the dynamic stall prediction. FlUENT's software is used for the numerical solution. Flow methodology for modelling 2-D unsteady viscous flow is presented and based on the incidence angle variation taken as a pulse using Gaussian function and oscillation forms. Results compared with experiment, show a very satisfactory agreement and highlight the need to take into account the unsteady flow and loads induced by movement of the structure.
\end{abstract}

Keywords: Wind turbine, Wind energy, Aerodynamics, Wind profile, Dynamic stall.

\section{NOMENCLATURE}

$\begin{array}{ll}\mathrm{C}_{\mathrm{L}} & \text { Lift force coefficient } \\ \mathrm{Cp} & \text { pressure coefficient } \\ \mathrm{Cy} & \text { force coefficient in the y direction } \\ \mathrm{c} & \text { chord } \\ \mathrm{dt} & \text { time step } \\ F y & Y \text { component of the resultant pressure } \\ & \begin{array}{l}\text { force acting on the lower side } \\ \text { generic functions }\end{array} \\ h, g & \text { height }\end{array}$

\section{INTRODUCTION}

The aerodynamic designer seeks an airfoil shape that achieves the best aerodynamic performance while taking into account the trade-offs with other disciplines and control. Significant progress has been made in developing computational methods for fluid flow analysis. An aerofoil section undergoes dynamic stall when is subjected to any form of unsteady angle of attack motion (pitching, plunging, etc.), which takes the effective angle of attack beyond its normal static stall angle. For developments concerning especially ship building applications thick profiles are also often used. The characteristics of these profiles are of high interest as far as applications involving high angles of incidence at which the performances of the profiles must be partly preserved. Wind Fluctuations in direction are so fast that it is impossible that the axis for a wind turbine be

\author{
i(t) time incidence angle \\ $i, j, k \quad$ space index \\ $k \quad$ kinetic turbulence energy \\ $u \quad$ absolute velocity \\ Greek \\ $\alpha \quad$ angle of attack \\ $\alpha_{\mathrm{m}} \quad$ amplitude of angle of attack \\ $\varepsilon \quad$ dissipation rate of $\mathrm{k}$ \\ $\omega$ dissipation rate frequency of $\mathrm{k}$
}

aligned continuously in wind direction because of the machine inertia. Therefore, the impact of the flow around the wing varies very rapidly for values that exceed the static stall angle of the airfoil. This variation may be causing the dynamic stall phenomenon. This phenomenon is due to the appearance of a strong or sudden flow separation on the suction side surface of a profile. This then causes a drastic drop in lift and increases drag and a reversal of the bending moment. Dynamic stall of an aerofoil is characterized by the shedding of a strong vertical disturbance from its leading edge, which is called a dynamic stall vortex. The onset of flow separation is also delayed to an angle of attack higher than the static stall angle and, combined with the effects of the dynamic stall vortex, is responsible for elevated values of lift. The wind turbine blades are also submitted in time to degradation in its performance due to aerodynamic surface roughness of 
its blades. These surface irregularities are caused by the accumulation of insect debris, ice or the operating live. The stall mechanism is highly studied, but it remains poorly understood. Experimental and numerical studies have analyzed the effects of the incidence variations and the dynamic stall and especially the airfoil movement in oscillation. A detailed review on the dynamic stall is provided by references (Kunz et al. 1992; Holmes 1988; Tulapurkara 1996) which provided a very detailed physical analysis of this phenomenon). The analysis of instantaneous changes of direction and speed of wind, such as those shown in Fig. 1 and Fig. 2 (see Le Gourieres 2008), show that the changes in direction exceeds 20 degrees in a very short time.

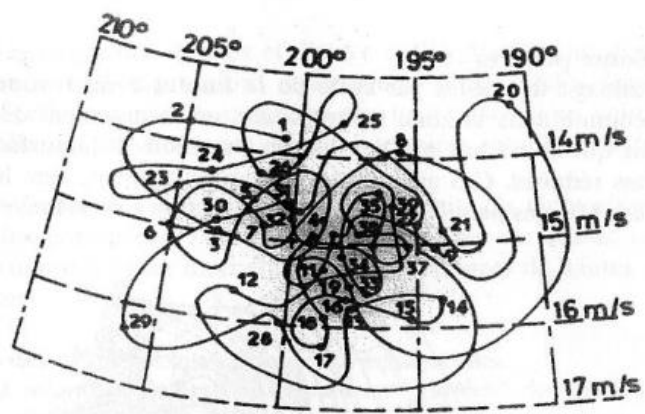

Fig. 1. Wind direction variation.

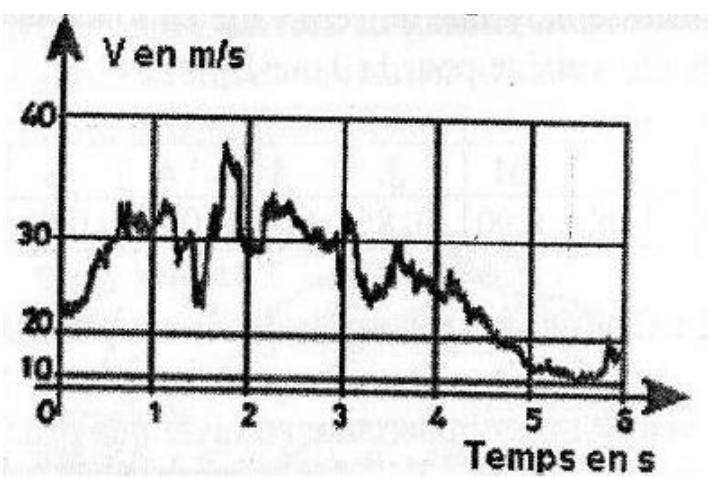

Fig. 2. Wind velocity variation.

Wind energy today has become an industrial power generation quite important in some countries. This renewable energy is the first objective of community and global issues arising from the aim of many countries to reduce $\mathrm{CO}_{2}$ gas emissions. Wind energy is therefore required in the coming years to achieve a significant development in the world. The methods of rotor dynamic machines design using two and threedimensional approaches form a tool in the engineering of such machines. The detailed analysis of the topology of the flow is indispensable for the study of various phenomena responsible for the aerodynamic losses. Many of the rotors found on current generation HAWT systems are designed using a combination of 2-D airfoil tools and three-dimensional blade element and momentum (BEM) theory. A number of comprehensive computer codes using this methodology are currently available to the designer. In these methods, unsteady flow effects are either ignored, or modelled using a synthesis of 2-D data. As a result, these methods are incapable of accurately modelling three-dimensional dynamic stall processes, tower shadow effects, tip relief effects, and sweep effects. These three-dimensional effects can alter the airloads, affect the fatigue life, and significantly influence the coast of ownership of HAWT systems.

The subcontractor shall adopt a mathematical and numerical formulation to analyze a flow field around a HAWT. The flow field is divided into three distinct entities: a thin viscous region surrounding the individual blades, a potential flow region that transfer the disturbances generated by the rotor to the far field, and a helical wake structure (vortices) that includes the tip vortices shed by the rotor blades. The study of horizontal axis wind HAWT operating in real wind conditions remains a very complex and difficult to be modelled. Fluctuations in wind direction are so fast that it is impossible for a wind turbine axis, to be aligned continuously in wind direction due to machine inertia. Therefore, the impact of the flow incidence angle around the wing varies very rapidly to reach values above the airfoil static stall angle. This variation is suspected to be causing the phenomenon of dynamic stall.

A computational research program shall be performed by Advanced Mechanics Laboratory of University of Science and Technology Houari Boumediene (USTHB), Algiers, Algeria, in the area of horizontal axis wind turbine (HAWT) aerodynamics. The research shall focus on (a) understanding the flow mechanisms that affect the performance of wind turbines to nonaxial and non-uniform inflow, and (b) the contribution in the development of modern, efficient computational techniques that complement existing combined blade element-momentum theory (Yous and Dizene 2008).

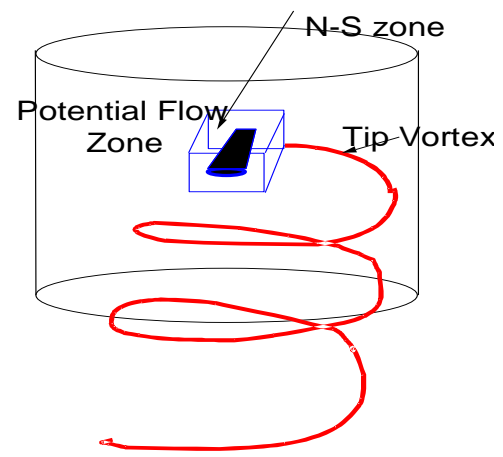

Fig. 3. The physics of the interaction between viscous regions, inviscid regions and vortices

The present work was motivated by the need to understand the physics of the interaction between viscous regions, inviscid regions and vortices, as shown in the Fig. 3 sketched above (Lakshmi et al. 2000). These regions are modelled using different methodologies, making the present approach of comparisons of two turbulence models with threedimensional calculations. Studies were done to assess the effects of two turbulence models on the predicted performance. A standard $\mathrm{k}-\varepsilon$ turbulence model (Launder 1974) and the model by Menter (1994). Three-dimensional Reynolds-Averaged Navier-Stokes equations are used for unsteady state flow and a Gaussian function and oscillation forms of incidence 
angle variations are applied as a flow methodology for modelling 2-D unsteady viscous flow. The dynamic stall is due to the appearance of a massive detachment of the flow on the upper surface of a profile subject to a critical incident or sudden. Consequently, this causes a sudden drop in lift, coupled with increased drag and a reversal of the bending moment. The stall mechanism is widely studied but remains not very understood. Most of studies have dealt with the flow around a rotor helicopter configuration stall. Many other experimental and numerical studies have analyzed the incidence variation effects on the dynamic stall but in oscillation airfoil configuration.

The variation of the incidence angle of the flow over and around an airfoil may be provided by the dynamic stall phenomenon. The intensity of this phenomenon requires the structures of wind turbines significant stresses that must be taken into account in the design of the structure. In general, it is possible to define the dynamic stall like an airfoil subjected to unsteady phenomena due to its own movement or a change in direction and amplitude of flow upstream velocity. This phenomenon occurs frequently in the context of an airfoil undergoing swinging for which the maximum angle of incidence exceeds the static stall angle of the profile. In these conditions, we observe the development of a hysteresis cycle on the drag and lift coefficients and on timing of oscillation. Many experimental studies have been conducted to analyze the effects of variation of the angle of incidence and the dynamic stall, considering the study of motion of an airfoil in oscillation. The experimental work of the Laboratory of Aerodynamics and Biomechanics of Movement (PL), led notably by using embedded laser anemometry has provided a very detailed physical analysis of this phenomenon (see the work of Croskey 1982 and Pizali 1993).

Recent studies of turbulent flows in operating stall were conducted; we can cite for example the references Berton (1997) and Autric (1994). The objective of this work contributes to the prediction of the dynamic stall phenomenon of a turbulent flow around a horizontal axis of wind turbine rotor by studying the 2D flow around a NACA0012 profile in a moving pulse and pitch oscillation. The URANS method is used for solving equations and turbulence modeling is provided by the k- $\varepsilon$ and k-SST $\omega$ models. In order to validate this approach a comparison of numerical results with experimental data found in the literature (Pizali 1993) has been made. The methodology adopted in this work is to begin by analyzing the physics of the unsteady flow around the wind turbine rotor subjected to wall rotation effects of. For this reason, and the purpose of a qualitative assessment of the turbulent flow characteristics, we undertook a simulation of the threedimensional unsteady flow.

\section{Governing EQUATIONS}

\subsection{Three Dimensional URANS Case}

All the flow equations are presented in Cartesian tensor notation. For a unsteady incompressible flow, the conservation laws of mass, momentum and energy may be as below. Since RANS and URANS are commonplace, details of these methodologies are not given here.

In RANS, only the time-averaged equations are solved, and the fluctuations associated as Reynolds stresses $\left(-\overline{u_{i}^{\prime} u_{j}^{\prime}}\right)$ are represented by turbulence models

Continuity and Momentum equations are

$$
\begin{aligned}
& \frac{\partial}{\partial x_{i}}\left(\tilde{U}_{i}\right)=0 \\
& \frac{\partial \tilde{U}_{i}}{\partial t}+\frac{\partial}{\partial x_{i}}\left(\tilde{U}_{i} \tilde{U}_{j}\right)=-\frac{1}{\rho} \frac{\partial \bar{P}}{\partial x_{i}}+\frac{\partial}{\partial x_{i}}\left(\bar{\tau}_{i j}-\overline{u_{i} u_{j}}\right) \\
& \bar{\tau}_{i j}=2 \mu\left(S_{i j}-\frac{1}{3} S_{k k} \delta_{i j}\right) \\
& \approx 2 \bar{\mu}\left(\tilde{S}_{i j}-\frac{1}{3} \tilde{S}_{k k} \delta_{i j}\right)
\end{aligned}
$$

\subsection{Two Dimensional URANS Simulation Case}

In order to assess qualitatively the dynamic stall effect on rotor aerodynamic performance, simulations in a two-dimensional configuration are performed. Pascazio (1995) showed that the transition point of the boundary layer is strongly unsteady and it may be possible during an oscillation cycle to see respectively a laminar boundary layer followed by a transition point and then finally fully turbulent boundary layer. The maximum incidence angle, in contrast to the Reynolds number, has a major effect on the dynamic stall. Several experimental studies were conducted, including those conducted by NASA (Allmaras 1992). Alister (1978) has shown the effect on the wind turbine performance, for a given frequency.

Simulations are performed using some of parameters governing the flow as: the Reynolds number, the oscillation frequency, the amplitude of the oscillation and the average angle of attack. These parameters are related by a sinusoidal variation law of the incidence angle given by:

$\alpha(\mathrm{t})=\alpha_{\mathrm{m}}+\mathrm{A} \sin (2 \mathrm{nft})$

The choice of values of three parameters that govern the flow is identical to the experimental data of test cases conducted by Mc Alister (1978). The average incidence angle $\alpha_{\mathrm{m}}=15$ degree and the amplitude of oscillation is 20 degree.

These values induce a maximum incidence angle of 25 degree. When the oscillation amplitude and the maximum angle of incidence become important, they induce a deep stall even if the reduced frequency of oscillation is relatively low.

\subsection{Turbulence Modeling}

Turbulence modelling remains a key challenge in CFD. In the present work, the modelled transport equations were solved using a three dimensional FLUENT CFD code. A number of different models based on the $\mathrm{k}-\varepsilon$ and the $\mathrm{k}-\omega$ closures were used in this study. The various models under the appropriate categories are listed below. 


\subsection{1 $k-\varepsilon$ Model}

In the $\mathrm{k}-\varepsilon$ model the Reynolds stress is modelled as:

$-\overline{\rho u_{i} u_{j}}=2 \mu_{t}\left(\tilde{S}_{i j}-\frac{1}{3} S_{k k} \delta_{i j}\right)-\frac{2}{3} \bar{\rho} K$

The eddy viscosity $\mu_{\mathrm{t}}$ is related to the turbulent kinetic energy $\mathrm{K}$ and to its dissipation rate $\varepsilon$ as:

$$
\mu_{t}=C_{\mu} \bar{\rho} \frac{K^{2}}{\varepsilon}
$$

The distribution of $\mathrm{k}$ and $\varepsilon$ in the flow field is determined from their modelled transport equations (Launder \& Spalding, 1974)

$$
\begin{aligned}
& \frac{\partial}{\partial x_{i}}\left(\bar{\rho} \tilde{U}_{i} K\right)=\frac{\partial}{\partial x_{i}}\left(\left(\mu+\frac{\mu_{t}}{\sigma_{K}}\right) \frac{\partial K}{\partial x_{i}}\right) \\
&+G-\bar{\rho} \varepsilon \\
& \frac{\partial}{\partial x_{i}}\left(\bar{\rho} \tilde{U}_{i} \varepsilon\right)= \frac{\partial}{\partial x_{i}}\left(\left(\mu+\frac{\mu_{t}}{\sigma_{\varepsilon}}\right) \frac{\partial \varepsilon}{\partial x_{i}}\right) \\
&+C_{\varepsilon 1} \frac{\varepsilon}{K} G-C_{\varepsilon 2} \bar{\rho} \frac{\varepsilon^{2}}{K} \\
& \mathrm{G}=\mu_{\mathrm{t}}\left(\frac{\partial \tilde{\mathrm{U}}_{\mathrm{i}}}{\partial \mathrm{x}_{\mathrm{j}}}+\frac{\partial \tilde{\mathrm{U}}_{\mathrm{j}}}{\partial \mathrm{x}_{\mathrm{i}}}\right) \frac{\partial \tilde{\mathrm{U}}_{\mathrm{i}}}{\partial \mathrm{x}_{\mathrm{j}}}
\end{aligned}
$$

The high-Re model avoids the need to integrate the modelled equations right down to the wall by making use of the universal behaviour near wall flows. The standard wall- function approach is:

Zero value at the wall for $\mathrm{K}$ and $\varepsilon=\frac{C_{\mu}^{3 / 4} K^{3 / 2}}{0,4 \delta y}$.

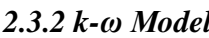

The low-Reynolds k- $\varepsilon$ models suffer from the lack of appropriate wall boundary condition for $\varepsilon$ which is specified mostly by ad-hoc empirical functions for the near wall flow. The choice of the specific dissipation rate $\omega$ is therefore sometimes preferred since the near wall $\omega$ behaviour is known and therefore the boundary condition at the wall can be specified more accurately. The transport equations for the $\mathrm{k}$ and $\omega$ have the following source terms:

$$
\begin{aligned}
& \frac{\partial}{\partial t}(\rho k)+\bar{u}_{j} \frac{\partial}{\partial x_{j}}(\rho k)=\tau_{i j} \frac{\partial \bar{u}}{\partial x_{j}}-\beta^{*} \rho \omega k \\
& +\frac{\partial}{\partial x_{j}}\left[\left(\mu+\sigma_{k} \mu_{t}\right) \frac{\partial k}{\partial x_{j}}\right] \\
& \frac{\partial}{\partial t}(\rho \omega)+\bar{u}_{j} \frac{\partial}{\partial x_{j}}(\rho \omega)=\frac{\mu}{\mu_{t}} \tau_{i j} \frac{\partial \bar{u}}{\partial x_{j}}-\beta \rho \omega^{2}+\frac{\partial}{\partial x_{j}}\left[\left(\mu+\sigma_{\omega} \mu_{t}\right) \frac{\partial \omega}{\partial x_{j}}\right] \\
& +2\left(1-F_{1}\right) \rho \sigma_{\omega 2} \frac{1}{\omega} \frac{\partial k}{\partial x_{i}} \frac{\partial \omega}{\partial x_{j}} \\
& S_{K}=P-\rho \beta^{*} \omega K
\end{aligned}
$$

$S_{\omega}=\alpha \frac{\omega}{K} P-\rho \beta \omega^{2}$

and where $\mu_{t}=\alpha^{*} \frac{K}{\omega}$

The constants of k- $\varepsilon$ and k- $\omega$ for SST model are given in below.

$\sigma_{\mathrm{k} 1}=0.85, \sigma_{\omega 1}=0.5, \beta_{1}=0.075, \mathrm{a}_{1}=0.31$,

$\beta^{*}=0.09, \mathrm{~K}=0.41, \gamma_{1}=\frac{\beta_{1}}{\beta^{*}}-\frac{\sigma_{\omega 1} K^{2}}{\sqrt{\beta^{*}}}$

$\sigma_{\mathrm{k} 2}=1.0, \sigma_{\omega 2}=0.856, \beta_{2}=0.0828, \beta^{*}=0.09$,

$\mathrm{K}=0.41$,

$\gamma_{1}=\frac{\beta_{2}}{\beta^{*}}-\frac{\sigma_{\omega 2} K^{2}}{\sqrt{\beta^{*}}}$

\section{NUMERICAL METHOD}

The computations presented in this paper have been obtained using a finite-volume FLUENT ccommercial computational fluid dynamic package. Velocity components, turbulent kinetic energy and its dissipation rate profiles for fully developed turbulent flow have been applied from leading edge to trailing edge past the airfoils. It has been necessary to perform some griddependence studies. Solution that seems to be independent from grid size has been obtained after four subsequent adaptations. The total size of grid after adapting is about 308 thousands computational cells.

\subsection{Three Dimensional Computational Domain Grid}

The dimensions of the computational domain are as follows: Due to the symmetry of a rotor-blade horizontal axis wind turbines, the computational domain is defined as a half-cylinder. The radius of the half-cylinder is equal to 12 times chord length and its height is equal to 8 times chord length. The mesh used is shown in Fig. 4 and is unstructured and consists of a single block. So, the NACA0012 blade profile is meshed in a block which is unstructured and consists of 28,866 triangular cells and 14,698 nodes. The blade movement is generated by the rotation of the domain and this method is known as moving Reference Frame.

All the governing equations with the imposed boundary conditions are solved using FIUENT solver Reynolds numbers of $10^{6}$. Once this first step done, several simulations were performed and compared to experimental results. The computational domain dimensions are of 10 times chord length height and 20 times chord length long spreaded on both of the profile sides of the rotation centre. Oscillation point is located one quarter of the chord length. The blade pulse movement is operated by the profile movement in a deformable mesh.. The flow calculations around the profile in stationary position and pitching motion are achieved using a spatial pattern of "third-order and for the unsteady patterns temporal implicit time step of two order. For the unsteady flow we have three parameters settings in terms of the Reynolds number and angle of attack. Those of oscillatory profile are the amplitude of motion, the average angle and frequency that will define different regimes of dynamic stall. The mesh 
fineness near the wall is $\Delta \mathrm{x}=10^{-4}$ which allows $\mathrm{y}+=0$, 30.

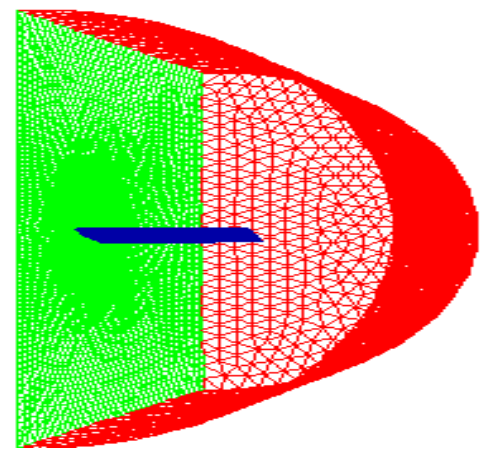

(a) Grid of the Computational domain.

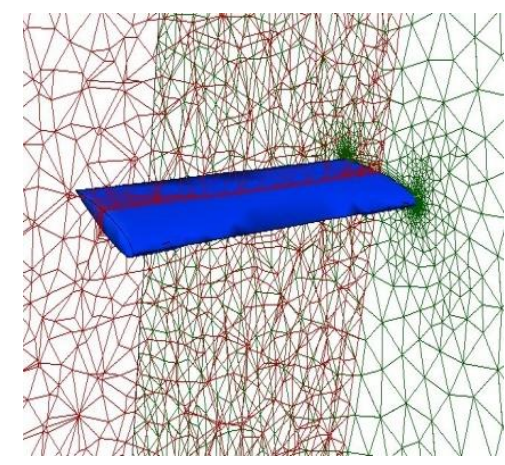

(b) Meshing refinement around the airfoil.

Fig. 4. Three Dimensional Computational grid and boundaries of NACA0012 airfoil profile.

The unsteady terms are implicit second-order discretized. A centered SIMPLE algorithm is used for the pressure-velocity coupling and a third-order MUSCL scheme discretization is used for the convection and diffusion terms. The conservation equations are solved using a segregated solver. The boundary conditions selected for this simulation are the velocity upstream and the static pressure downstream. The movement of the blade was managed by en bloc movement of the entire mesh method by reference moving (moving reference frame). The calculations are performed for a Reynolds number above $0.6 .10^{6}$. The three rotation speeds of the blade are simulated as: a zero angular velocity; $10 \mathrm{rd} / \mathrm{s}$ and $30 \mathrm{rd} / \mathrm{s}$ angular velocities.

\subsection{Two Dimensional Computational Domain Grid}

The dimensions of the 2D computational domain are as follows: a height of 10 times chord length, a 20 times chord length long where 10 times chord length upstream from the center of rotation of the profile, located at one quarter of the chord and 10 times chord length downstream. The upstream Reynolds number used for these simulations is $10^{6}$. The numerical scheme is offset upstream space of order 3 and the numerical scheme is a dual time step of order 3 . The turbulence models are the $\mathrm{k}-\varepsilon$ and $\mathrm{k}-\mathrm{SST} / \omega$. The mesh is unstructured and the airfoil movement in the computational domain is generated by a deformable grid. Figure 5 shows this grid and it has 14,926 nodes.

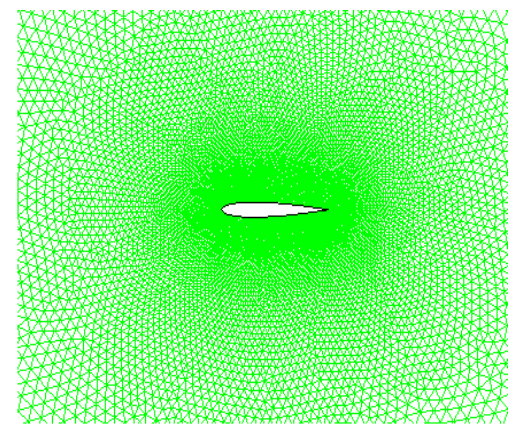

(a) Computational grid domain.

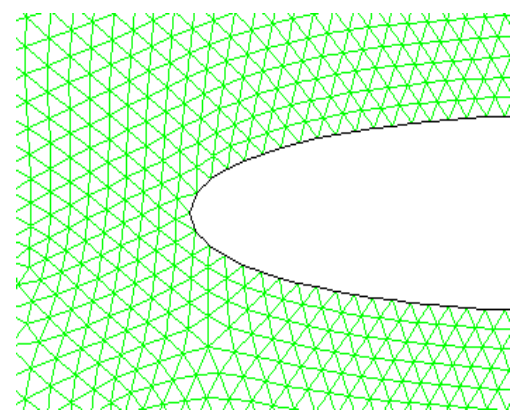

(b) Meshing refinement around the airfoil L.E.

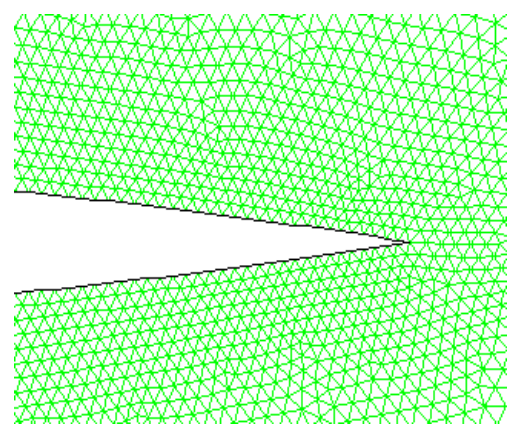

(c) Meshing refinement around the airfoil T.E.

Fig. 5. Two Dimensional Computational grid and boundaries of NACA0012 airfoil profile.

\section{RESULTS AND DISCUSSION}

The calculation results presented in this work are related to flow behaviour around the NACA 0012 profile. The unsteady three-dimensional results of constant velocity contours at a swig phase and backward phase are presented and discussed using the k- $\omega$ SST models. A number of $2 \mathrm{D}$ calculations have been carried for the NACA 0012 airfoil at unsteady state and at various angles of attack for a period of oscillation.

\subsection{Unsteady Three Dimensional Computations}

The three dimensional flow and the visualisation were calculated in the symmetrical plane and presented on Figure 6 at low subsonic regime. 


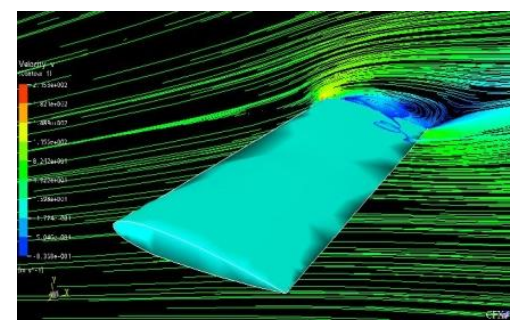

(a) Three Dimensional separation flow and vortices SST model

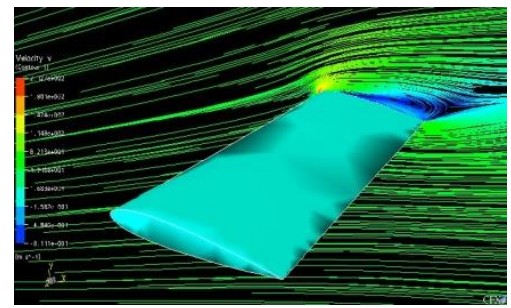

(b) Three Dimensional separation flow and vortices $\mathrm{k}-\varepsilon$ model

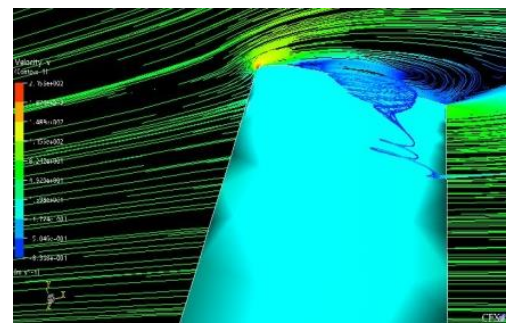

(c) Three Dimensional flow (zoom) SST model

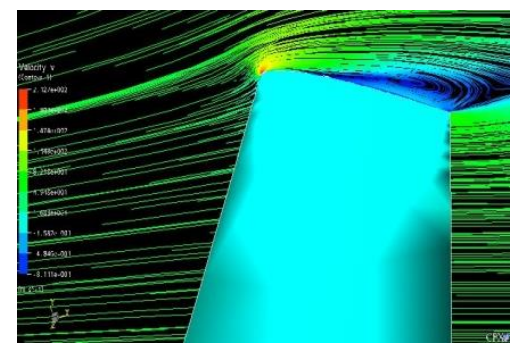

(d) Three Dimensional flow (zoom) k- $\varepsilon$ model

Fig. 6. Computations past NACA0012 profile. Flow separation and vortices development in symmetrical plane. Comparison between SST and k- $\varepsilon$ models

We can observe the differences between the SST model and the $\mathrm{k}-\varepsilon$ model in term of streamlines of flow development. Here, the SST model seems to better predict and resolves the flow separation and the passage vortex structure better on the suction side. At the symmetrical plane located as a fixed beam downstream of the blade leading edge (Figures $6 a, 6 b, 6 c$ and $6 d$ ), the SST calculations results show the vortex structure in stall flow configuration. The phenomena is very sensitive to the model of turbulence and the eddy viscosity do not suppress the onset and size of the recirculation zone, more in the case of SST model. The flow calculations around rotating profiles are made using patterns in discrete space not shifted upstream of the first order and patterns in discrete time implicit dual-type of the three order.

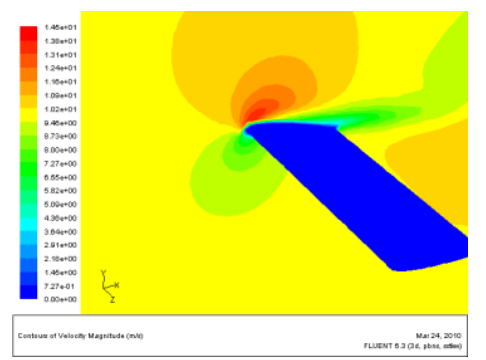

(a) Angular Velocity $=0 \mathrm{rd} / \mathrm{s}$, K- $\omega$ SST .

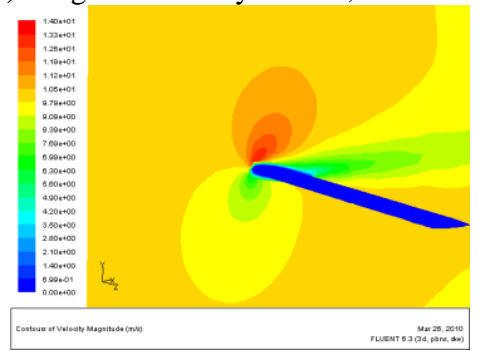

(b) Angular Velocity $=0 \mathrm{rd} / \mathrm{s}, \mathrm{K}-\varepsilon$.

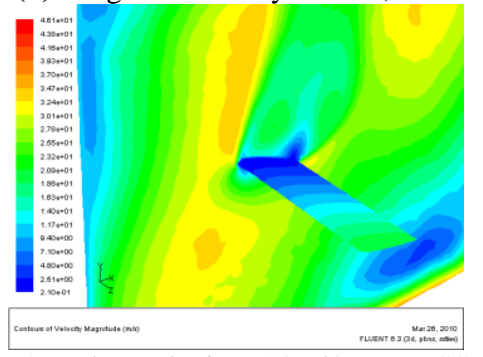

(c) Angular Velocity $=10 \mathrm{rd} / \mathrm{s}, \mathrm{K}-\omega \mathrm{SST}$.

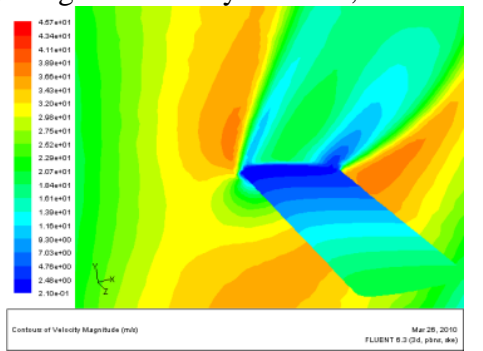

(d) Angular Velocity $=10 \mathrm{rd} / \mathrm{s}, \mathrm{K}-\varepsilon$.

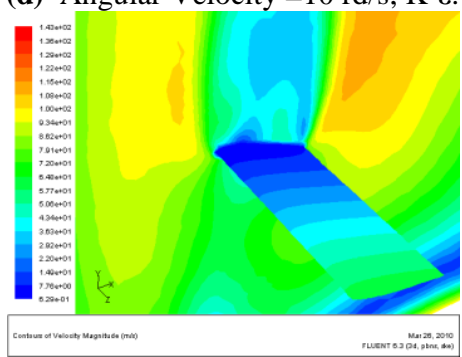

(e) Angular Velocity $=30 \mathrm{rd} / \mathrm{s}, \mathrm{K}-\omega \mathrm{SST}$.

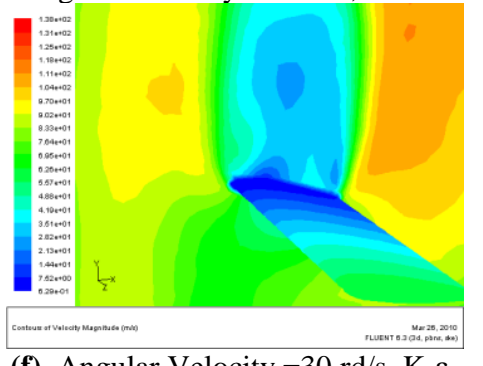

(f) Angular Velocity $=30 \mathrm{rd} / \mathrm{s}, \mathrm{K}-\varepsilon$.

Fig. 7. Velocity Profiles versus angular speed $\omega$ around NACA0012 profile. 
The blade rotation is generated by a moving block of the total grid using the moving reference frame. All calculations are performed for a Reynolds number of 0.6.10 $0^{6}$ and for three rotation velocities of the blade, namely a zero velocity, equal to $10 \mathrm{rd} / \mathrm{s}$ and equal to $30 \mathrm{rd} / \mathrm{s}$. The objective of numerical simulation in this case is to represent the unsteady flow conditions. The level of unsteadiness is determined by the reduced frequency and is greater than 0.1 , which corresponds to an unsteady flow. The initial blade incidence angle is 15 degree, which represents a value that must be greater than the static stall angle of the profile.

Figure 7 shows the values of the constant velocity of three-dimensional turbulent flow. The prediction of the turbulence model $\mathrm{k}-\omega \mathrm{SST}$, at zero velocity of the blade, shows a flow separation as we move beyond the static stall angle around a blade of 15 degree as shown in Fig. 7a. The flow becomes unsteady when the blade rotates at a speed of $10 \mathrm{~d} / \mathrm{s}$, so it becomes fully separated around the pall (see Fig. 7c) under the effect of centrifugal force. Indeed it is for this reason that uses twisted wing to reduce the effects of tip vortex structures due to the rotation of the blade causing the dynamic stall phenomenon. This separation flow becomes more important as shown in the Fig. 7e when velocity increases to $30 \mathrm{rd} / \mathrm{s}$. The comparison of results obtained using the two turbulence models do not show significant differences in the evolution of the unsteadiness of the flow when the blade is rotating except at the tip blade where there are some differences between the flows.

\subsection{Unsteady Two Dimensional Computations}

Hysteresis cycles observed on the lift coefficient are presented in Fig. 8. The k- $\varepsilon$ model seems to appear less efficient than the model $k-\omega$ SST compared with the experiments.

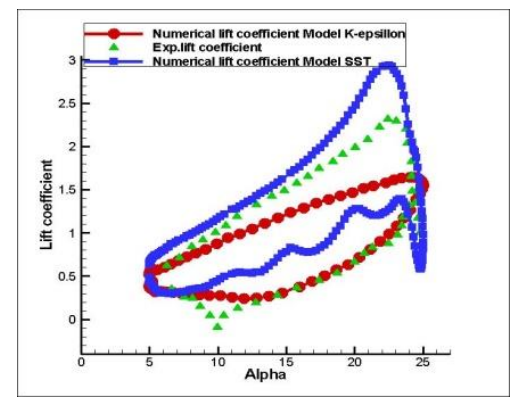

Fig. 8. Hysteresis cycles of lift coefficient computations; Comparison with experiment

Maximum and minimum values have the same order of magnitude as those of the experiment. However, the phenomenon of dynamic stall is governed by the end of the upward movement of the profile. The development of leading edge vortex on the entire suction surface of the profile appears delayed compared with experimental data. This behavior is characteristic of URANS models which were calibrated according to the hypothesis of a boundary layer without adverse pressure gradient. The model tends to overestimate the turbulence viscosity and therefore underestimate the adverse pressure gradient responsible for the separation of the boundary layer and therefore stall. The shape of the hysteresis cycle obtained and the angles of incidence for which the profile will stall during the upward movement and then reattach during the downward movement will be dependent on the two combined effects (the adverse pressure gradient due to the angle of incidence and the circulation induced by the movement of the profile due to the angular velocity profile), depending on the phase of the cycle.

The pressure coefficient $\mathrm{Cp}$ is computed in the same conditions as the velocity field, i.e. for a period of oscillation and shown in Fig. 9. We observe negative values on the suction side surface during the upward phase flow. This large depression is located at a distance of $30 \%$ chord from the leading edge for $\alpha=$ 16.8 degree and grows toward the trailing edge at angle of attack of 24.8 degree. During the downward phase corresponding to angle of $\alpha$ of 24.5, 23.6, 20.6 and 11.9 degrees, the flow seems to reattach the suction side surface. These observations show that the risk of dynamic stall will take place probably during the upward phase of the oscillation.

The behavior of $\mathrm{Cp}$ variations here is in agreement with the one of Mc Alister test case. However, the amplitude of oscillation and the angle of incidence means being more important, we observe that the vortex shedding from the leading edge, which represents the trigger phase of the stalled movement, occurs before the end of the upward phase, with an angle of incidence of about 22.7 degree.

\section{ConClusion}

The mechanism of dynamic stall is influenced by a combination of complex and interdependent physical phenomena. In fact, according to analysis of the problem physics, it is shown that just before the dynamic stall angle value, we observe that the transition laminar / turbulent usually occurs near the leading edge in a thin laminar layer or a bulb of laminar separation which is of very small size of the order of $1 \%$ chord. Modeling approach of this phenomenon was made in this work, based on two-Dimensional and threeDimensional unsteady URANS computations. Comparison of simulation results between the models $\mathrm{k}-\varepsilon$ and $\mathrm{k}-\omega / \mathrm{SST}$ shows a good ability of k-omega model to predict the start-time of dynamic stall due to its calibration for the boundary layer. The time at which the vortices are released leading edge and trailing edge is well captured by this model. When the incidence angle is important, while being greater than the static stall angle, the lift failure at the stall will be brutal. However, there is an overestimation of the lift and strong oscillations in the lift coefficient of lift during the descending phase. It also notes that the instant at which the hydrofoil is occupied by the leading edge vortex appears to be predicted earlier than in the experimental data. There is also the development of vortices on the upper surface of the profile that are not apparent in the experiment. The flow around a profile by upward phase is very special case. The addition of a two-dimensional geometry and the forcing through the oscillation makes a strong two-dimensional flow. 


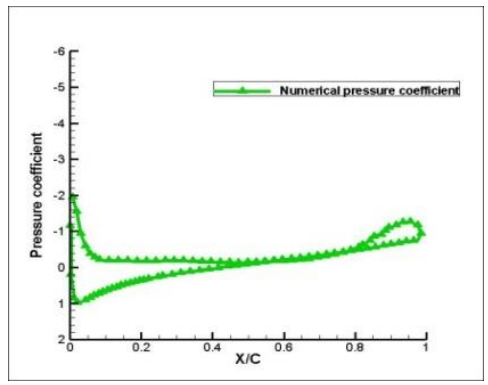

(a) $\alpha=8.9$ degree upward phase, K- $\omega$ SST.

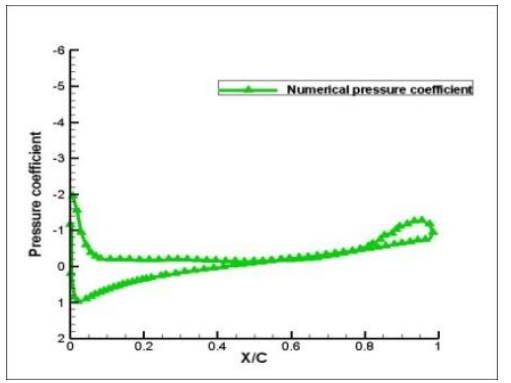

(b) $\alpha=13.1$ degree upward phase, K- $\omega$ SST.

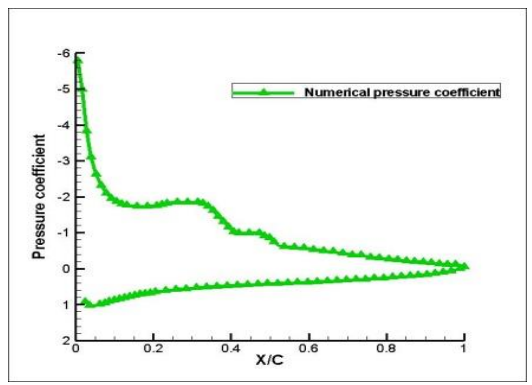

(c) $\alpha=16.8$ degree upward phase, K- $\omega$ SST.

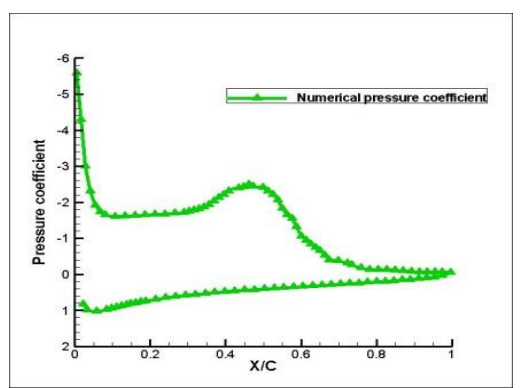

(d) $\alpha=19.8$ degree upward phase, K- $\omega$ SST.

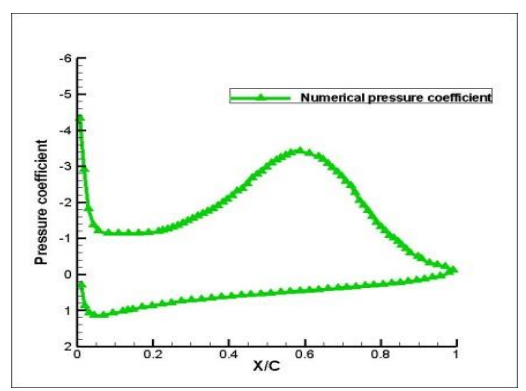

(e) $\alpha=22.7$ degree upward phase, K- $\omega$ SST.

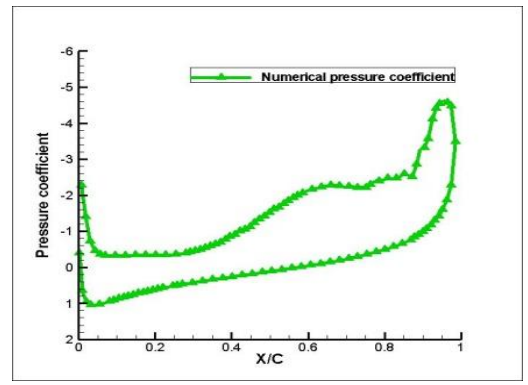

(f) $\alpha=24.8$ degree upward phase, K- $\omega$ SST.

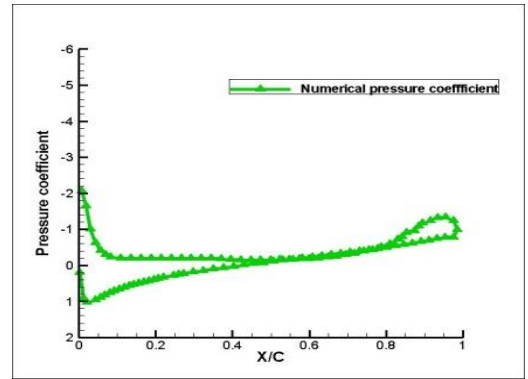

(g) $\alpha=24.5$ degree downward phase, K- $\omega$ SST.

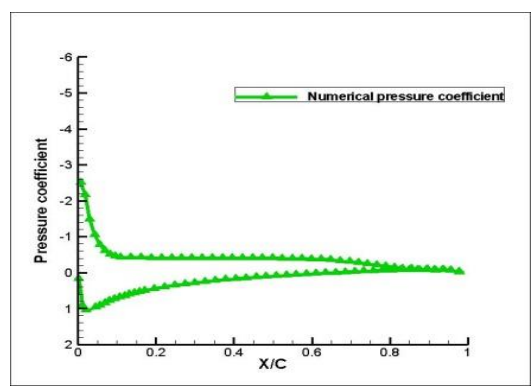

(h) $\alpha=23.6$ degree downward phase,. K- $\omega$ SST.

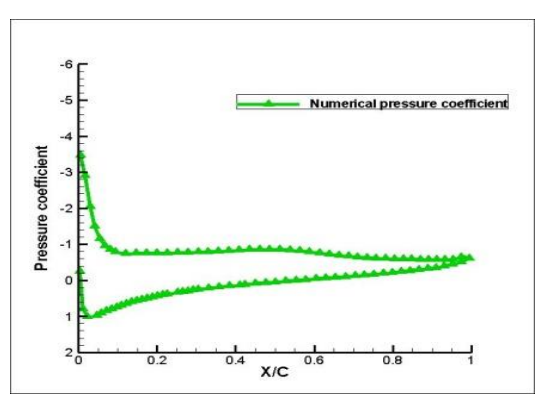

(i) $\alpha=20.6$ degree downward phase, K- $\omega$ SST.

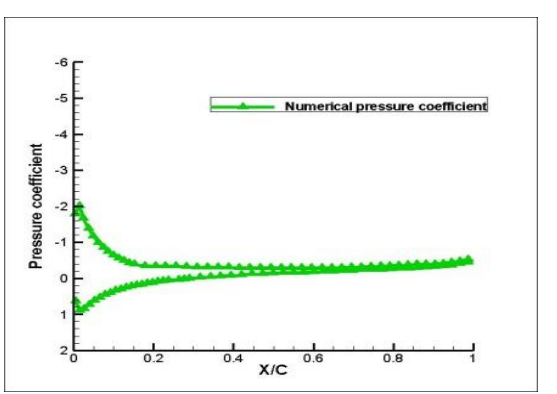

(j) $\alpha=11.9$ degree downward phase, K- $\omega$ SST.

Fig. 9. Pressure Coefficient variations for one oscillation period. K- $\omega$ /SST turbulence model. 
Vortex shedding from the leading edge, which represents the trigger phase of the stalled movement, is observed to occur before the end of the upward phase, with an angle of incidence of about 22.7 degree.

\section{ACKNOWLEDGEMENTS}

I would like to thank Dr. Sofiane Khelladi for his contribution in writing this paper and for welcoming me in his team. I also thank Dr. Massouh for welcoming me in his Aerodynamics Laboratory of Arts ET Métiers School in Paris Tech., France.

\section{REFERENCES}

Allmaras, S.R. and P.R. Spalart (1992). A one equation turbulence model for aerodynamics flows. AIAA paper, 92-0439.

Autric, J.M. (1994). Contribution à l'étude expérimentale et numérique du développement dela couche limite sur un modèle oscillant : la transition en écoulement instationnaire. thesis, Laboratoire d'Aérodynamique et de Biomécanique du Mouvement (LABM), Université Aix Marseille II, France.

Berton, E., D. Favier and M. Maresca (1997, June). Embedded L.V. methodology for boundary-layer measurements on oscillating models. AIAA, Proceedings of the 28th Fluid Dynamics Conf., AIAA paper $97 / 1832$, Snowmass.

Holmes, D.G. and S.D. Connell (1988). Solution of 2-D Navier-Stokes Equations using a k- $\varepsilon$ Turbulence model. Flygtekniska Forsoksanstalten TN-198819, Stockholm, Sweden.

Kunz, R.F. and B. Lakshminarayana (1992). Explicit Navier-Stokes computation of cascade flows using the k- $\varepsilon$ turbulence model. AIAA Journal 30(1).

Lakshmi, N., P.Tu. Sankar and W. Wolfe (2000). Computational studies of horizontal axis wind turbines. School of Aerospace Engineering Georgia Institute of Technology.

Launder, B.E. and B.I. Sharma (1984). Application of the energy dissipation model of turbulence to the calculation of flow near a spinning disc. Letters in Heat and Mass Transfer I(2), 131-138.

Le Gourieres, D. (2008). Les eoliennes. Théorie, conception et calcul pratique. D. LE GOURIERES, Editions du moulin cadiou.

Mc Alister, K.W., L.W. Carr and W.J. Mc Croskey. (1978). Dynamic stall experiments on the NACA0012 airfoil. Technical Paper TP 1100, NASA.

McCroskey, W.J. (1982). Unsteady airfoils. Annual Review of Fluid Mechanics, 14.
Menter, F.R. (1994). Two-equation eddy-viscosity turbulence models for engineering applications. AIAA-Journal 32(8).

Pascazio, M. (1995). Contribution expérimentale à l'étude de la couche limite se développant sur un profil d'aile en oscillation : phénomènes de transition et de décollement en écoulement instationnaire. Thèse de dotorat, Université de la Méditerranée (Aix-Marseille II).

Piziali, R.A. (1993). An experimental investigation of 2D end 3D oscillating wing aerodynamics for a range of angle of attack including stall. NASA technical memorandum, $N^{\circ}$ 4632. Ames, $C A$ :NASA 4632, Chalmers University of Technology.

Tulapurkara, E.G. (1996). Turbulence models for the computation of flow past airplanes. PII: S03760421(96) 00002-4.

Yous, I. and R. Dizene (2008, April). Reynolds Averaged Navier-Stokes Equations Simulation of Horizontal Axis Wind Turbine Rotor Dynamic Stall Response. $N^{\circ}$ spécial de la Revue des Energies Renouvelables, CDER; Proceedings of CICME 2008, Sousse Tunisia. 